\section{Abstracts of papers presented at the 28th Genetic Society's Mammalian Genetics and Development Workshop held at the UCL Great Ormond Street Institute of Child Health, University College London on Thursday 30th November 2017}

Edited by: NICHOLAS D. E. GREENE ${ }^{1}$, ANDREW J. COPP ${ }^{1}$ AND CYNTHIA L. ANDONIADOU ${ }^{2}$

${ }^{1}$ UCL Great Ormond Street Institute of Child Health, 30 Guilford Street, London, WC1N 1EH

${ }^{2}$ KCL Craniofacial Development and Stem Cell Biology, Floor 27 Tower Wing, Guy's Campus SE1 9RT

Identifying Novel Genes in Human and Mouse Optic Fissure Closure.

AARA PATEL ${ }^{1}, \begin{aligned} & \text { J. HAYWARD } \\ & \text { DAHLMANN-NOOR }\end{aligned}{ }^{1}$,
ANNEGRET
YASSIR ABOU-RAYYAH ${ }^{2,3}$ C. GABRIEL ${ }^{3}$,
L. JENKINS ${ }^{4}$ AND JANE C. SOWDEN ${ }^{1}$
${ }^{1}$ Stem Cells and Regenerative Medicine Section UCL
GOS-ICH
${ }^{2}$ Moorfields Eye Hospital
${ }^{3}$ Great Ormond Street Hospital for Children
${ }^{4}$ North Thames Regional Molecular Genetics
Laboratory, GOSH

Ocular coloboma, is a congenital eye malformation often causing severe visual impairment. It results from a defect in optic fissure closure, an essential process during early eye morphogenesis, and may occur together with small eyes (microphthalmos). Changes in cell shape and extracellular matrix are observed during the process of closure, but the genetic pathways regulating these are not well understood. Human molecular genetic studies to date have identified over 80 genes that when mutated cause microphthalmia, anophthalmia, and/or coloboma (MAC). Our analysis of 89 children with MAC indicates that many cases are not caused by mutation of these known genes. To better understand the molecular pathology of these eye malformations we conducted transcriptome analysis of genes expressed as the optic fissure closes in embryogenesis. We used laser capture microdissection to isolate tissue from the margins of the closing optic fissure in human and mouse embryonic eyes. Transcriptome analysis identified sets of genes, some conserved in both species, which are strongly and transiently expressed in the closing optic fissure. A bioinformatics analysis of these genes showed that several of them are involved in regulating cell shape and extracellular matrix remodelling making them candidates for regulating the process of closure.
Characterisation of Retinal Degeneration in Idh3a Mutant Mice

\section{AMY S. FINDLAY, KLARA NOVAKOVA, LISA MCKIE, MARGARET KEIGHREN, RODERICK CARTER, BECKY STARBUCK, NICK MORTON, PAUL POTTER AND IAN J. JACKSON \\ MRC Human Genetics Unit, IGMM, University of Edinburgh}

Isocitrate dehydrogenase (IDH) is an enzyme required for the production of $\alpha$-ketoglutarate from isocitrate, and is a tetramer made up of two $\alpha$, a $\beta$ and a $\gamma$ subunit. Loss of function and missense mutations in IDH $3 \mathrm{~A}$ have previously been implicated in families exhibiting retinal degeneration. We identified mice with late-onset retinal degeneration in a screen of ageing mice carrying ENU-induced mutations. Low-resolution mapping followed by whole genome sequencing found a missense mutation in the $\alpha$-subunit gene, Idh $3 a(\mathrm{E} 229 \mathrm{~K})$. Mice homozygous for this mutation, exhibit signs of retinal stressas early as 3 months and photoreceptor degeneration from 7 months. We used CRISPR/Cas9 technology to produce a knockout of $I d h 3 a$ and find that homozygous mice do not survive past early embryogenesis. Idh $3 \mathrm{a}^{\mathrm{E} 229 . \mathrm{K} / \mathrm{-}}$ compound heterozygous mutants exhibit a more severe retinal degeneration, showing severely deteriorated ERG responses by 2 months, accompanied by photoreceptor loss and GFAP staining. Analysis of mitochondrial function on MEF mutant cell lines highlighted a reduction in both mitochondrial maximal respiration and reserve capacity levels in both $\operatorname{Idh} 3 a^{E 229 K /}$ ${ }_{E 229 K}$ and $I d h 3 a^{E 229 K /-}$ cells. It has been previously reported that the retina operates with a limited mitochondrial reserve capacity and we suggest that this, in combination with the reduced reserve capacity in mutants, explains the degenerative phenotype observed. 

Microtia as a common feature of hearing loss
syndromes

JUAN M. FONS ROMERO AND ABIGAIL S. TUCKER

Centre for craniofacial and stem cell biology, King's College London, Guy's hospital

Several human syndromes are characterized by hearing loss with mutations affecting the development of the three ear components. Microtia is a common feature with variable severity, classified as grade I, II, III and IV (Anotia). Examples of these syndromes are Branchio-oto-renal syndrome, due to Eya1 mutations, DiGeorge syndrome, being Tbx 1 mutations the main cause, and LADD syndrome, due to FGF10 mutations. We aim to uncover the genetic interactions between Eya1, Tbx1 and FGF10 during auricle development. We found that Eyal is expressed in the mesodermally-derived auricle muscle, downstream of Tbx1. Loss of function of either gene led to a lack of cartilage due to a downregulation of Sox9 in the ectomesenchyme and to a lack of auricle muscle differentiation, as shown by the downregulation of MyoD. Interestingly, Sox9 deletion does not interfere with auricle development. FGF10 mutant mice display a mild microtia, characteristic of LADD syndrome. We observed that FGF10 mutant auricles are arrested during development and that loss of FGF10 does not affect Sox9 expression. We propose a model in which Tbx1/Eyal positive mesodermal muscle instructs the ectomesenchyme to induce auricle development and to differentiate into cartilage by upregulation of Sox9.

Folate and methionine metabolism is essential for neural tube closure

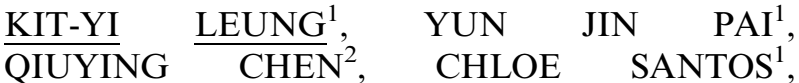
SONIA SUDIWALA ${ }^{1}$, STEVEN S. GROSS ${ }^{2}$, ANDREW J. COPP ${ }^{1}$ AND NICHOLAS D. E. GREENE ${ }^{1}$

'Developmental Biology \& Cancer Programme, UCL Great Ormond Street Institute of Child Health, University College London, London, WCIN 1EH, UK ${ }^{2}$ Department of Pharmacology, Weill Cornell Medical College of Cornell University, 1300 York Avenue, New York, NY 10021, USA

Abnormal folate one-carbon metabolism (FOCM) is implicated in neural tube defects (NTDs), severe malformations of the nervous system. MTHFR mediates unidirectional transfer of methyl groups from the folate cycle to the methionine cycle and therefore represents a key nexus in partitioning one-carbon units between FOCM functional outputs. Methionine cycle inhibitors prevent neural tube closure in mouse embryos. Similarly, inability to use glycine as a onecarbon donor to the folate cycle causes NTDs in glycine decarboxylase (Gldc) deficient embryos. However, analysis of Mthfr null mouse embryos shows that neither s-adenosylmethionine abundance nor neural tube closure depend on one-carbon units derived from embryonic or maternal folate cycles. Mthfr deletion or methionine treatment prevent NTDs in Gldc null embryos by retention of onecarbon units within the folate cycle. Overall, neural tube closure depends on activity of both the methionine and folate cycles but transfer of one-carbon units between the cycles is not necessary.

PLC $\zeta$ is the physiological trigger of the $\mathrm{Ca}^{2+}$ oscillations that induce embryogenesis in mammals but conception can occur in its absence.

ALAA HACHEM ${ }^{1}$, J. GODWIN ${ }^{2}$, M. RUAS ${ }^{1}$, H.C. LEE $^{3}, \quad$ M. FERRER BUITRAGO ${ }^{4}$, G. ARDESTANI ${ }^{3}$, A. BASSETT ${ }^{5}$, S. FOX $^{1}$, F. NAVARRETE ${ }^{3}$ P. DE SUTTER $^{4}$, B. HEINDRYCKX ${ }^{4}$ R. FISSORE $^{3}$ AND J. PARRINGTON ${ }^{1}$

Department of Pharmacology, University of Oxford, Mansfield Road, Oxford OX1 3QT, UK

${ }^{2}$ Department of Biochemistry, University of Oxford, South Parks Road, Oxford OXI 3QU, UK

${ }^{3}$ Department of Veterinary and Animal Sciences, University of Massachusetts, 661 North Pleasant Street, Amherst, MA 01003-9286, USA

${ }^{4}$ Department for Reproductive Medicine, Ghent University Hospital, De Pintelaan 185, 9000 Ghent, Belgium

${ }^{5}$ Sir William Dunn School of Pathology, University of Oxford, South Parks Road, Oxford OX1 3RE, UK

Activation of the egg by the sperm is the first, vital stage of embryogenesis. The sperm protein PLC $\zeta$ has been proposed as the physiological agent that triggers the $\mathrm{Ca}^{2+}$ oscillations that initiate embryogenesis. Consistent with this, recombinant PLC $\zeta$ induces $\mathrm{Ca}^{2+}$ oscillations in eggs and debilitating mutations in the PLCZ1 gene are associated with infertility in men. However, there has been no evidence that knockout of the gene encoding PLC $\zeta$ abolishes the ability of sperm to induce $\mathrm{Ca}^{2+}$ oscillations in eggs. Here, we show that sperm derived from Plcz1 ${ }^{-/-}$male mice fail to trigger $\mathrm{Ca}^{2+}$ oscillations in eggs, cause polyspermy and thus demonstrate that PLC $\zeta$ is the physiological trigger of these $\mathrm{Ca}^{2+}$ oscillations. Remarkably, some eggs fertilized by PLC $\zeta$-null sperm can develop, albeit at greatly reduced efficiency, and after a significant time-delay. In addition, Plcz1 ${ }^{-1-}$ males are subfertile but not sterile, suggesting that in the absence of 
$\operatorname{PLC} \zeta$, spontaneous egg activation can eventually occur via an alternative route. This is the first demonstration that in vivo fertilization without the normal physiological trigger of egg activation can result in offspring. PLC $\zeta$-null sperm now make it possible to resolve longstanding questions in fertilization biology, and to test the efficacy and safety of procedures used to treat human infertility.

MLL2 conveys transcription-independent H3K4me3 in the oocyte

COURTNEY W. HANNA ${ }^{1,2}$, AARON TAUDT ${ }^{3,4}$, JIAHAO HUANG ${ }^{1}$, LENKA GAHUROVA ${ }^{5,6}$, ANDREA KRANZ ${ }^{7}$, SIMON ANDREWS ${ }^{8}$, WENDY DEAN ${ }^{1}$, FRANCIS A. STEWART ${ }^{7}$, MARIA COLOMÉ-TATCHÉ ${ }^{3,4}$ AND GAVIN KELSEY ${ }^{1,2}$

'Epigenetics Programme, Babraham Institute, Cambridge, CB22 3AT, UK

${ }^{2}$ Centre for Trophoblast Research, University of Cambridge, Cambridge, CB2 3EG, UK

${ }^{3}$ Institute of Computational Biology, Helmholtz Zentrum München, Ingolstädter Landstr. 1, Neuherberg 85764, Germany

${ }^{4}$ European Research Institute for the Biology of Ageing, University of Groningen, University Medical Center Groningen, A. Deusinglaan 1, Groningen $9713 \mathrm{AV}$, The Netherlands

${ }^{5}$ University of South Bohemia, Ceske Budejovice, 37005, Czech Republic

${ }^{6}$ Institute of Animal Physiology and Genetics, ASCR, Libechov, Czech Republic

Biotechnology Center TU Dresden, Tatzberg, 01307, Germany

${ }^{8}$ Bioinformatics Group, Babraham Institute, Cambridge, CB22 3AT, UK

Trimethylation of histone 3 lysine 4 (H3K4me3) is classically thought of as a mark of active promoters and yet it occurs at untranscribed domains. Partial redundancy of $\mathrm{H} 3 \mathrm{~K} 4$ methyltransferases has made it difficult to delineate the mechanisms underlying genomic targeting of $\mathrm{H} 3 \mathrm{~K} 4 \mathrm{me}$. The oocyte provides an attractive system to investigate this, because extensive acquisition of $\mathrm{H} 3 \mathrm{~K} 4 \mathrm{me} 3$ occurs in a non-dividing cell and ablation of a single $\mathrm{H} 3 \mathrm{~K} 4$ methyltransferase, Mll2, prevents most H3K4me3. We developed low-input chromatin immunoprecipitation to interrogate promoter-associated histone modifications $\mathrm{H} 3 \mathrm{~K} 4 \mathrm{me} 3$, H3K27ac and H3K27me 3 throughout oogenesis. In non-growing oocytes, $\mathrm{H} 3 \mathrm{~K} 4 \mathrm{me} 3$ was restricted to transcriptionally active promoters, but as oogenesis progresses, H3K4me3 accumulates in a transcription-independent manner: targeted to broad inter-genic regions, putative enhancers, and transcriptionally silent H3K27me3- marked promoters. Consequently, thousands of bivalent domains are established during oogenesis. Ablation of Mll2 resulted in loss of transcriptionindependent $\mathrm{H} 3 \mathrm{~K} 4 \mathrm{me} 3$, with limited effects on transcription-coupled $\mathrm{H} 3 \mathrm{~K} 4 \mathrm{me} 3$ or gene expression. Deletion of Dnmt3alb showed that DNA methylation protects regions from acquiring $\mathrm{H} 3 \mathrm{~K} 4 \mathrm{me} 3$. Our findings show that there are two independent mechanisms of targeting $\mathrm{H} 3 \mathrm{~K} 4 \mathrm{me} 3$ to genomic elements, with MLL2 recruited to unmethylated $\mathrm{CpG}$-rich regions independently of transcription.

\section{The epigenetic regulation of mouse cerebellar development}

KIMBERLEY. L. H. RIEGMAN $^{1}$, CHARLOTTE GEORGE ${ }^{2}$, CONOR MOHAN $^{1}$, DAVID SIMS $^{2}$, CAMERON OSBORNE ${ }^{3}$ AND M. ALBERT BASSON $^{1}$

'King's College London, Centre for Craniofacial and Regenerative Biology, Floor 27 Tower Wing, Guy's Hospital, London, SE1 9RT, UK

${ }^{2}$ University of Oxford, MRC Computational Genomics Analysis and Training Programme (CGAT), MRC Centre for Computational Biology, MRC Weatherall Institute of Molecular Medicine, John Radcliffe Hospital, Headington, Oxford, OX3 9DS, UK

${ }^{3}$ King's College London, Department of Genetics \& Molecular Medicine, Floor 8 Tower Wing, Guy's Hospital, London, SE1 9RT, UK

Hypoplasia of the cerebellum is a neurological condition that has been associated with a range of motor and emotive symptoms, often severely impacting on the patient's quality of life. In many cases the underlying genetic cause of this condition is unknown. We hypothesise that some of the disease-causing mutations are located in regulatory regions of the genome. To identify putative regulatory elements that may control cerebellar growth we performed promotor capture $\mathrm{Hi}-\mathrm{C}$ (PCHi-C) in primary cerebellar granule cell precursors (GCps). This experiment revealed 77,820 unique promoter-interacting regions. Several genomic regions that interact with the promoters of genes implicated in cerebellar development and/or hypoplasia were identified. Our group has previously demonstrated that the chromatin remodeller CHD7 is critical for the proliferation of GCps. To identify how CHD7 regulates the chromatin landscape in GCps, we performed ATACseq. This analysis demonstrated that CHD7 functions primarily to maintain an "open" chromatin state. Many of these regions overlap with/or are in the vicinity of promoter-interacting regions identified in PCHi-C. Data illustrating how we can use information about putative regulatory elements identified by PCHi-C and genome-wide 
chromatin accessibility to infer locations of to-date unannotated regulatory elements, some of which are regulated by CHD7, will be discussed.

\section{Humanising the mouse Tardbp gene}

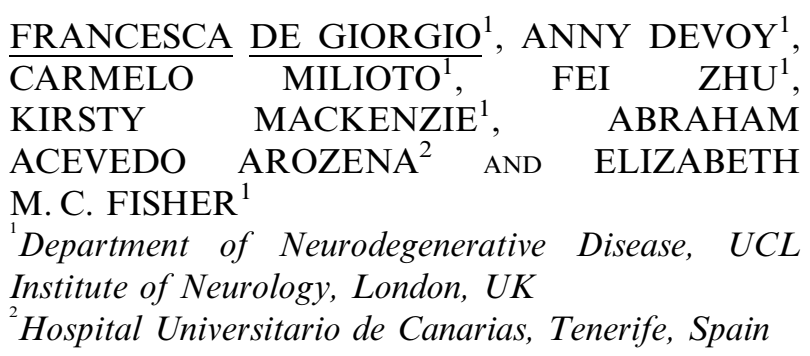

Amyotrophic lateral sclerosis is a neurodegenerative disease characterised by degeneration of upper and lower motor neurons showing protein aggregates in the cytoplasm. Mutations in the TARDBP gene, encoding the TAR DNA-binding protein (TDP-43), are causative for ALS. Depletion of wild type TDP-43 from the nucleus with aggregates of ubiquitinated/hyperphosphorylated TDP-43 in the cytoplasm is a common feature for both sporadic and familial ALS cases (Ling et al., 2015). TDP-43 is a RNA/DNA binding protein, mainly located in the nucleus and involved in multiple molecular mechanisms (Scotter et al., 2015). Thus, there is an urgent need to develop accurate animal models to better understand the role of TDP-43 in ALS. We are creating a knock-in humanised TDP-43 mouse, replacing the genomic region of mouse Tardbp from the ATG codon until the stop codon, including introns, with the corresponding sequence from the human orthologous TARDBP. Using human/mouse Bacterial Artificial Chromosomes (BACs) as sources of DNA sequences to perform BAC recombineering and homologous recombination, we will target mouse embryonic stem cells generating a mouse carrying a fully genomically humanised TDP-43 with mouse untranslated regions. Our humanised mouse may become an important tool for future studies on human ALS caused by TDP-43.

\section{Developing Models of Paediatric Low Grade Glioma}

\section{JULIE E COOPER, SCOTT HASTON AND JUAN PEDRO MARTINEZ-BARBERA}

UCL Institute of Child Health, 30 Guilford Street, London WCIN IEH

Paediatric Low Grade Glioma (pLGG) is the most common childhood brain tumour, and while its prognosis is generally good (being slow growing and nonmalignant), it is less favourable in recurrent or inaccessible tumours. Genetic analysis of pLGG indicates it is a single pathway disease - the most common somatic mutations are activating rearrangements and mutations of $B$-Raf, followed by FGFRI and others, all resulting in an over-activation of the Mitogen Activated Protein Kinase (MAPK) pathway. This MAPK activation leads to an initial proliferative phase of tumour formation (likely a developmental process) which is followed by a secondary senescent phase. Our aim is to generate genetically engineered mouse models of pLGG with which to elucidate the etiology of the disease and to conduct preclinical pharmacological trials. We have generated a model in which the oncogenic B-RAF ${ }^{\mathrm{v} 600 \mathrm{e}}$ activating mutation is conditionally expressed in Hesxl-expressing cells of the anterior brain early in development. These mice develop proliferative lesions prenatally, resembling ectopic stem cell compartments, which may recapitulate the proliferative phase of $\mathrm{pLGG}$ formation. Targeting the B-RAF ${ }^{\mathrm{v} 600 \mathrm{e}}$ activating mutation to Sox2-expressing neuroepithelium during the expansion phase of neural development replicates the lesions, indicating these cells to be the cell-oforigin. Unfortunately neither of these models is compatible with postnatal survival so we are investigating the postnatal fate of the lesions to determine whether they represent a true model of pLGG, using ex vivo culture and in utero electroporation.

Regulation of YAP/TAZ in the pituitary is necessary
for normal development and postnatal homeostasis

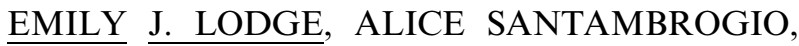
JOHN P. RUSSELL AND CYNTHIA L. ANDONIADOU

Centre for Craniofacial and Regenerative Biology, King's College London, UK

The pituitary gland is a major regulator of physiological processes such as growth, the stress response, fertility and metabolism. Deregulation of cell numbers either during development or postnatal life, can lead to hypopituitarism or tumours. Multiple signalling pathways are known to regulate pituitary development however the Hippo-YAP/TAZ cascade, known to regulate organ size, has not been investigated. Previously we have shown that key Hippo pathway components are expressed in the embryonic murine pituitary and expression persists in SOX2+ stem cells (Lodge et al., 2016). Here we show that a pituitary-specific deletion of Lats1 and Lats2 $\left(\right.$ Hes $x 1^{\text {Crel+ }} ;$ Lats $^{\text {flfl }} ;$ Lats $\left.^{\text {flff }}\right)$ in the developing pituitary leads to over-proliferation of the SOX2+ progenitor compartment and failure of cells to terminally differentiate. To determine if this phenotype is mediated through YAP, we employed a TetOn system to overexpress constitutive active YAP 
$\left(\right.$ Hesxl $\left.{ }^{\mathrm{Crel+}} ; \mathrm{R}^{\mathrm{rtTA}^{\text {th+ }}} ; \mathrm{Collal}^{\mathrm{Yapl-TetO} /+}\right)$, which led to an expansion of the embryonic SOX2+ compartment. While the pituitary-specific double LATS1/2 knockout is perinatal lethal, loss of LATS1 or one additional copy of LATS2 (HesxI ${ }^{\text {Crel+}^{+}}$; Lats ${ }^{\text {flf }} ;$ Lats $^{\text {fll+ }}{ }^{+}$) is sufficient to lead to a postnatal-onset phenotype comprised of pituitary tumours. These are characterised by accumulation of YAP and TAZ and poor differentiation; histopathological analyses suggests that these most closely resemble squamous pituitary carcinomas.

\section{Identifying the key function of $\mathrm{MeCP2}$ via genetic manipulation in mice}

REBEKAH $^{\text {TILLOTSON }}{ }^{1}$, JIM SELFRIDGE ${ }^{1}$, MARTHA V. KOERNER ${ }^{1}$, KAMAL K.E. GADALLA ${ }^{2,3}$, JACKY GUY ${ }^{1}$, DINA DE SOUSA $^{1}$, RALPH D. HECTOR ${ }^{2}$, STUART R. COBB $^{2}$ AND ADRIAN BIRD ${ }^{1}$

${ }^{1}$ The Wellcome Centre for Cell Biology, University of Edinburgh, Michael Swann Building, King's Buildings, Max Born Crescent, Edinburgh EH9 3BF, UK

${ }^{2}$ Institute of Neuroscience and Psychology, College of Medical, Veterinary and Life Sciences, University of Glasgow, Glasgow G12 8QQ, UK

${ }^{3}$ Pharmacology Department, Faculty of Medicine, Tanta University, Tanta 31527, Egypt

Mutations in the X-linked MECP2 gene cause the profound neurological disorder, Rett syndrome (RTT), which affects 1 in 10-15,000 girls. MeCP2 protein is an epigenetic reader, binding to $\mathrm{mCG}$ and mCAC sites in the genome. How it interprets the DNA methylome, however, remains elusive. Several mechanisms have been proposed, including a role in transcriptional repression, activation, alternative splicing and miRNA processing. As a result, $\mathrm{MeCP} 2$ is often referred to as a 'multifunctional' protein. At odds with this view, missense mutations that cause RTT are concentrated in two discrete clusters coinciding with interaction sites for partner macromolecules: the Methyl-CpG Binding Domain (MBD) and the NCoR/SMRT Interaction Domain (NID). We therefore hypothesised that the single dominant function of $\mathrm{MeCP} 2$ is to recruit the NCoR/SMRT co-repressor complex to chromatin. We have tested this by producing and characterising knock-in mice expressing mutant forms of $\mathrm{MeCP} 2$ that lack sequences outside these two domains. We then explored the therapeutic potential of a shortened allele - consisting of just the MBD and the NID. We show that, despite high evolutionary conservation, regions of $\mathrm{MeCP} 2$ are functionally dispensable. Excitingly, this finding could be beneficial for gene therapy as shortening the coding sequence creates greater capacity for regulatory sequences.
Underlying molecular mechanism involved in blood vessel migration during tooth development

HIBA ASRAR AND ABIGAIL TUCKER Centre for Craniofacial and Stem Cell Biology, King's College University of London, Guy's Hospital Tower, Floor 27, London SE1 9RT, UK

Blood vessels play an important part in regulating tooth development, facilitating essential gas exchange, delivering vital nutrients and growth factors to the dental apparatus. Research has suggested that the emerging vasculature also plays an instructive role in controlling morphogenesis. WNT signalling has proven to regulate blood vessel formation in other systems. Moreover, Vascular Endothelial Growth Factor (VEGF) is known to be a potent mediator of angiogenesis. Angiogenesis in other systems has been shown to be modulated by chemo-repellents, known as Semaphorins (Bates, Taylor et al. 2003). Semaphorins are axon guiding molecules but they indirectly contribute to angiogenesis by chemo repelling endothelial cells. We hypothesized that WNTs control blood vessel migration by inducing semaphorin expression and that blood vessels are providing signalling cues to differentiating cells during tooth development. In situ hybridization to detect Sem3f expression revealed that inhibiting Wnts downregulated semaphorin $3 \mathrm{~F}$ in the mouse molar, suggesting that WNTS control endothelial cell migration in the dental papilla by regulating Semaphorins. Disrupting blood vessel migration by VEGF inhibitor led to a differentiation blockade of the pre-secretory ameloblasts and odontoblasts. These results conclude the importance of the developing vasculature and provide insight that will aid in establishing a functionally viable bioengineered tooth.

\section{Molecular basis for early embryonic lethality in Parp1/2 null embryos}

RUSLAN STROGANTSEV $^{1}$, CLAIRE SENNER ${ }^{1}$, WENDY DEAN $^{1}$, DOMINIC SPENSBERGER ${ }^{2}$ AND MYRIAM HEMBERGER ${ }^{1}$

${ }^{1}$ Epigenetics ISP, Babraham Institute, Cambridge, CB22 $3 A T$

${ }^{2}$ Gene Targeting Facility, Babraham Institute, Cambridge, CB22 $3 A T$

Parp1 together with its closely related Parp2 catalyse poly ADP-ribosylation of their target proteins and play important roles in DNA damage response as well as chromatin remodelling and transcriptional regulation. Mouse models of Parp1 and Parp2 deficiency show increased sensitivity to DNA damaging agents and ionizing radiation, whilst double knock out (dKO) mice are early embryonic lethal. Here we investigate the molecular basis for lethality observed 
in Parp1/2 dKO embryos, using conditional Parp1 deletion mouse model. Consistent with previous reports, we find that whilst single Parp1 and Parp2 homozygous $\mathrm{KO}$ mice are viable, the combined deletion results in fully penetrant phenotype. Embryo analysis revealed E7.5 as the latest developmental stage when morphologically normal $\mathrm{dKO}$ conceptuses can be recovered, whilst severe abnormalities seen thereafter. In order to elucidate molecular mechanism for Parp1/2 dKO lethality we performed RNA-seq analysis identifying 1000 mis-regulated genes at E7.5 prior to any observable phenotype. Downregulated genes include key mediators of Wnt signalling as well as several developmentally regulated transcription factors involved in cellular differentiation, morphogenesis and embryo patterning. Intriguingly, we observe aberrant upregulation of core trophoblast stem cell - specific genes in the epiblast compartment as well as several imprinted genes with high placental expression. Taken together this indicates that Parp1 and Parp2 are likely to be involved in transcriptional regulation and/or epigenetic resetting occurring during gastrulation.

The Isolated Congenital Asplenia Gene, RPSA is required for rRNA Processing and Spleen Development.

JOHN N. GRIFFIN ${ }^{1,3}$, ANDREW ROBSON ${ }^{1}$, SAMUEL B. SONDALLE ${ }^{2}$, EMILY K. MIS ${ }^{1}$, GERALD GRIFFIN ${ }^{1}$, SUSAN J. BASERGA ${ }^{2,3}$ AND MUSTAFA K. KHOKHA ${ }^{1}$

Program in Vertebrate Developmental Biology, Departments of Pediatrics and Genetics, Yale University School of Medicine, 333 Cedar Street, New Haven, Connecticut 06510, USA

${ }^{2}$ Departments of Genetics, Molecular Biophysics and Biochemistry, and Therapeutic Radiology, Yale University School of Medicine, 333 Cedar Street, New Haven, Connecticut 06510, USA

${ }^{3}$ Centre for Craniofacial and Regenerative Biology, King's College London, London SE1 9RT, United Kingdom

Defects in the ubiquitous process of ribosome production are associated with a growing number of inherited disorders. Recently, mutations in RPSA were discovered to underlie approximately half of all Isolated Congenital Asplenia cases. However, the mechanisms by which lesions in this ribosome biogenesis factor lead specifically to spleen agenesis remain mysterious. Here we reveal that RPSA is required for normal spleen development in the frog Xenopus. Depletion of RPSA disrupts rRNA processing and ribosome biogenesis, and impairs expression of the key spleen patterning genes $n k \times 2.5$ and bapxl, specifically in the spleen anlage. Importantly, we also show that while injection of WT human RPSA RNA can recuse both rRNA processing and spleen patterning, RNA carrying a common disease variant cannot. Together, we present the first animal model of RPSA mediated isolated congenital asplenia, and reveal a crucial requirement for RPSA in rRNA processing and molecular patterning of the spleen during vertebrate development.

Genomic sequencing and reverse genetic approaches characterize C11orf70 gene: a novel player in the assembly of motor dyneins

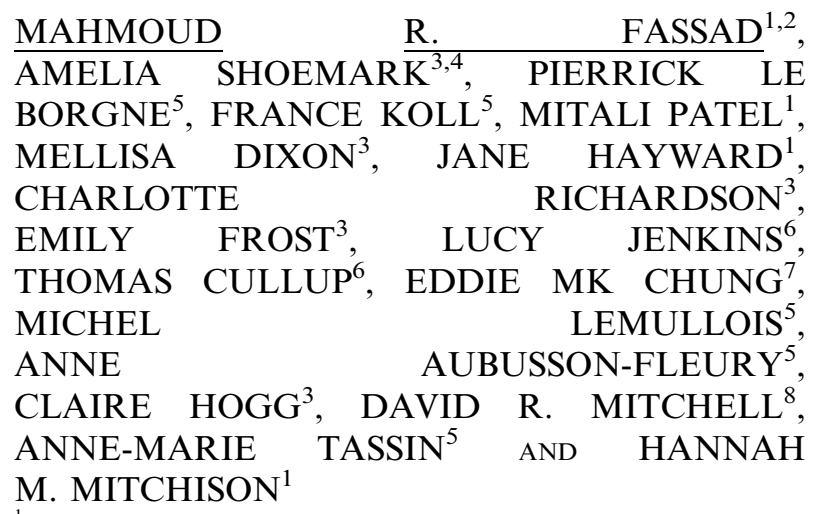

${ }^{1}$ Genetics and Genomic Medicine Programme, University College London, UCL Great Ormond Street Institute of Child Health, London WC1N $1 E H, U K$

${ }^{2}$ Department of Human Genetics, Medical Research Institute, Alexandria University, 165 El-Horreya Avenue El- Hadra 21561, Alexandria, Egypt

${ }^{3}$ PCD Diagnostic Team and Department of Paediatric Respiratory Medicine, Royal Brompton and Harefield NHS Trust, London SW3 6NP, UK

${ }^{4}$ School of Medicine, University of Dundee, Dundee, UK

${ }^{5}$ Institute for Integrative Biology of the Cell (I2BC), CEA, CNRS, Univ. Paris Sud, Université Paris-Saclay, 1 Avenue de la Terrasse, 91198 Gif sur Yvette, France

${ }^{6}$ Regional Molecular Genetics Laboratory, Great Ormond Street Hospital for Children NHS Foundation Trust, Queen Square, London WC1N $3 B H, U K$

${ }^{7}$ Population, Policy and Practice Programme, University College London, UCL Great Ormond Street Institute of Child Health, London WC1N $1 E H, U K$

${ }^{8}$ Department of Cell and Developmental Biology, SUNY Upstate Medical University, 750 E. Adams St., Syracuse, NY 13210, USA

Primary ciliary dyskinesia (PCD) is a rare genetic disease caused by defective cilia motility. It is characterized by chronic upper and lower respiratory problems associated with situs abnormalities and infertility. 
PCD causing mutations have been identified in more than 35 genes representing about $70 \%$ of affected individuals therefore new causative genes remain to be discovered. We use targeted next generation sequencing panels including all known PCD genes and other potential candidates for genetic diagnosis of PCD patients and novel gene discovery. We identified bi-allelic mutations in an uncharacterized gene, C1 1 orf70, in two unrelated families. All patients have a characteristic PCD phenotype with total immotility of respiratory cilia and inner and outer dynein arms (IDA + ODA) defect. C11 lorf70 is highly conserved across species with motile cilia and has a phylogenetic distribution similar to proteins associated with intraflagellar (IFT)- dependant dynein arms assembly. RNAi silencing of the Paramecium C11orf70 ortholog led to defective cilia motility and Paramecium swimming velocity with combined loss of IDA + ODA, a similar phenotype to the patients'. Further localization studies in Paramecium and Chlamydomonas showed that C11orf70 is mainly cytoplasmic protein with a tiny IFT-dependant flagellar distribution. These results support a role for C11 orf70 in the IFT-dependant assembly of ciliary dynein complexes.

Aggresomes inhibit multiple functions of the centrosome

ANILA IQBAL ${ }^{1}$, JENNIFER N. MURDOCH ${ }^{1}$, ANGELEEN FLEMING ${ }^{2}$ AND CHRISTOPHER J. WILKINSON ${ }^{1}$
'Centre for Biomedical Sciences, Royal Holloway University of London

${ }^{2}$ Department for Physiology, Development and Neuroscience, University of Cambridge

A hallmark of Parkinson's disease is the presence of Lewy bodies in the neurons of patients. These are large intracellular aggregates of many proteins but the largest constituent is alpha synuclein. Loss of sense of smell is associated with both Parkinson's and Alzheimer's, and may precede the other symptoms that lead to diagnosis. It is not known why the olfactory system is particularly vulnerable to damage. In the ciliopathy group of diseases, loss of cilia is known to cause anosmia (loss of smell) due to the odorant receptors being housed in the cilia of olfactory neurons. Using zebrafish larvae as a model, we report how the olfactory cilia needed for smell cannot be formed in the presence of protein aggregates similar to the Lewy Bodies found in Parkinson's disease. Furthermore, when such aggregated proteins are present, dopaminergic neurons cannot form cilia and are unable to organise their microtubule network. Both could contribute to loss of dopaminergic function and neuronal cell death in Parkinson's disease. Several neurodegenerative diseases, in which accumulation of misfolded proteins underlies disease development, may therefore be cilium diseases, opening up new routes for diagnosis and treatment. 